\title{
Effects of vacancies on overshooting in nonequilibrium ordering processes
}

\section{Gilhøj, Henriette; Jeppesen, Claus; Mouritsen, Ole G.}

Published in:

Physical Review E. Statistical, Nonlinear, and Soft Matter Physics

Link to article, DOI:

10.1103/PhysRevE.53.5491

Publication date:

1996

Document Version

Publisher's PDF, also known as Version of record

Link back to DTU Orbit

Citation (APA):

Gilhøj, H., Jeppesen, C., \& Mouritsen, O. G. (1996). Effects of vacancies on overshooting in nonequilibrium ordering processes. Physical Review E. Statistical, Nonlinear, and Soft Matter Physics, 53(5), 5491-5494. https://doi.org/10.1103/PhysRevE.53.5491

\section{General rights}

Copyright and moral rights for the publications made accessible in the public portal are retained by the authors and/or other copyright owners and it is a condition of accessing publications that users recognise and abide by the legal requirements associated with these rights.

- Users may download and print one copy of any publication from the public portal for the purpose of private study or research.

- You may not further distribute the material or use it for any profit-making activity or commercial gain

- You may freely distribute the URL identifying the publication in the public portal 


\title{
Effects of vacancies on overshooting in nonequilibrium ordering processes
}

\author{
Henriette Gilh $\not \mathrm{j},{ }^{1}$ Claus Jeppesen, ${ }^{2}$ and Ole G. Mouritsen ${ }^{1}$ \\ ${ }^{1}$ Department of Physical Chemistry, The Technical University of Denmark, Building 206, DK-2800 Lyngby, Denmark \\ ${ }^{2}$ Materials Research Laboratory, University of California, Santa Barbara, California 93106
}

(Received 31 October 1995)

\begin{abstract}
The effects of annealed site dilution on the nonequilibrium ordering process in the two-dimensional Ising model with a nonconserved order parameter have been studied using Monte Carlo simulation. It is found that the transient development of a local order that is larger than the equilibrium order (overshooting), as recently reported in the pure Ising model [H. Gilhøj, C. Jeppesen, and O. G. Mouritsen, Phys. Rev. Lett. 75, 3305 (1995)], persists in the dilute model and is accompanied by a depletion of the vacancies within the ordered domains. [S1063-651X(96)11505-1]
\end{abstract}

PACS number(s): 05.70.Ln, 64.60.My, 64.60.Cn, 75.40.Mg

Most model studies of nonequilibrium ordering processes following a sudden quench in temperature have focused on either the very early stage, where linearized theories can be invoked, or the late stage where asymptotic scaling laws and concepts of universality apply $[1,2]$. Less attention has been paid to the intermediate time regime where several length scales may be important corresponding to different types of ordering. In general, there are at least two different length scales in this regime, one being the average linear size of the ordering domain and the other being the correlation length of the fluctuations within the ordered domains. The latter length scale reflects the degree of local, average domain order.

Lately, evidence has accumulated that, whereas the average domain size is an increasing function of time, the local domain order is a concave function of time exhibiting a maximum at intermediate times. This maximum appears above the equilibrium value of the global order parameter. This phenomenon, called overshooting, has been observed in several computer simulation studies of different simple statistical-mechanical lattice models [3-5] as well as in experiments [6]. Overshooting has been hypothesized to be a consequence of a subtle competition between nonequilibrium enthalpy and nonequilibrium entropy as the system tries to lower its global free energy. The domain enthalpy, being a local system property, is minimized very quickly, whereas the entropy, which is a global measure, only become maximized over much longer time scales. The domain enthalpy, and consequently the domain order, overshoot their respective equilibrium values. Analogously, this phenomenon may be described as a transient accumulation of disorder in the network of interfaces bounding the ordered domains.

This present paper elaborates on this hypothesis by studying the effects of diffusing vacancies on the overshooting phenomenon in the Ising model when quenching into a onephase ordered region of the phase diagram. In comparison with the pure system, the dilution implies an additional contribution, the entropy of mixing, to the total system entropy. The dilution percentage is chosen to be so low that the phase to which the quench is performed is a one-phase state. The ordering process can now be described quantitatively by three different real-space measures as defined below: the average domain size, the average domain order parameter, and the average vacancy concentration in the domains.
The results are obtained from Monte Carlo computersimulation calculations performed on a simple lattice model, the two-dimensional ferromagnetic Ising model on a triangular lattice with annealed vacancies. The model is defined by the Hamiltonian

$$
\mathscr{H}=-J \sum_{\langle i, j\rangle} n_{i} n_{j} \sigma_{i} \sigma_{j}
$$

where $J>0, \sigma_{i}= \pm 1, n_{i}=0,1$ is a site occupation variable, and the summation is over nearest-neighbor sites. The lattice contains $N$ lattice points and is subject to periodic boundary conditions.

The vacancy concentration, $\rho=\left\langle 1-1 / N \Sigma_{i} n_{i}\right\rangle$, is kept fixed during the simulations and only a single vacancy concentration, $\rho=0.01$, has been considered. This concentration is sufficiently low to ensure that the Ising-type critical point is sustained although slightly suppressed in temperature [7]. The phase diagram for the diluted Ising model has a line of continuous transitions from the pure system critical point at $k_{B} T_{c} / J=2 / \ln 3 \simeq 1.82$ and ending in a tricritical point. This line separates a dilute ferromagnetic phase from a dilute disordered phase. Below the tricritical point there is a broad region of phase separation between a dilute ferromagnetic phase and a vacancy-rich disordered phase.

The microscopic dynamics used in the simulations is single-spin-flip (Glauber) dynamics for the Ising spins along with Kawasaki two-site exchange between vacancies and spins. The two types of dynamics are assumed to be independent and are for simplicity taken to occur on the same characteristic time scale. The actual choice of time scales is not qualitatively important for the results to be presented. The use of Glauber dynamics for the Ising spins automatically implies that the global order parameter, in this case the magnetization given by $\Psi=\left\langle N^{-1} \sum_{i} n_{i} \sigma_{i}\right\rangle$, is a nonconserved field. As the acceptance criterion for the Monte Carlo simulation, the usual Metropolis algorithm, $\min \left\{1, \exp \left(-\Delta \mathscr{H} / k_{B} T\right)\right\}$, is chosen, where $\Delta \mathscr{H}$ is the change in total internal energy associated with the attempted update. Time is measured in Monte Carlo steps (MCS) per lattice site. The effects of finite size have been assessed by using three different system sizes, $N=200^{2}, N=500^{2}$, and $N=1000^{2}$. The system is prepared at infinite temperature, 
corresponding to a completely random state, and then instantaneously quenched to a temperature below the orderdisorder transition line. With a quench temperature of $T / T_{c}=0.8$ and a vacancy concentration of $\rho=0.01$, the system is quenched into a one-phase state, the dilute ferromagnetic phase.

The temporal evolution of the order in the system is then monitored in three different ways as mentioned above. To account for the macroscopic order, the linear size, $R_{E}(t)$, of the domain has been calculated using the fact that, according to scaling and provided that the domain boundaries are localized, the total length of the boundary network, i.e., $\sim R_{E}(t)^{-1}$, is inversely proportional to the excess energy [8]. Hence

$$
R_{E}(t)=\langle\mathscr{H}\rangle_{T} /\left[\overline{\mathscr{H}(t)}-\langle\mathscr{H}\rangle_{T}\right],
$$

where $\langle\mathscr{H}\rangle_{T}$ is the internal energy at temperature $T$, and $\overline{\mathscr{H}(t)}$ is the ensemble average energy at time $t$ during the quench. These values have been obtained by averaging over a fairly large ensemble, i.e., many quenches (typically in the range 5-50, depending on system size).

We are, in particular, interested in the temporal evolution of the local ordering, i.e., the order within the domains. An ordered domain is defined as a set of lattice points spanned by a nearest-neighbor connected cluster of spins in the same spin state. We have defined a time-dependent local, average domain order parameter, $\Psi_{\text {loc }}(t)$, according to the following rules: (i) Only domains, labeled $k$, of size (area) $N_{k}$ beyond a certain minimum size, $N_{+}$, are included in the averaging. (ii) Clusters in these domains that are larger than a certain lower size, $N_{-}$, are considered as separate domains. (iii) The contributing domains are weighted according to their area. Apart from these restrictions, the definition of $\Psi_{\text {loc }}(t)$ is equivalent to the usual definition of the global order parameter (the magnetization), and can formally be written in the form

$$
\Psi_{\text {loc }}(t)=\overline{\left(\sum_{k}^{M} N_{k}\right)^{-1} \sum_{k=1}^{M}\left|\sum_{i_{k}=1}^{N_{k}} n_{i_{k}} \sigma_{i_{k}}\right|},
$$

where $M$ is the number of separate ordered domains larger than $N_{+}$found in a given configuration. The bar in Eq. (3) signifies an ensemble average. We have verified that the

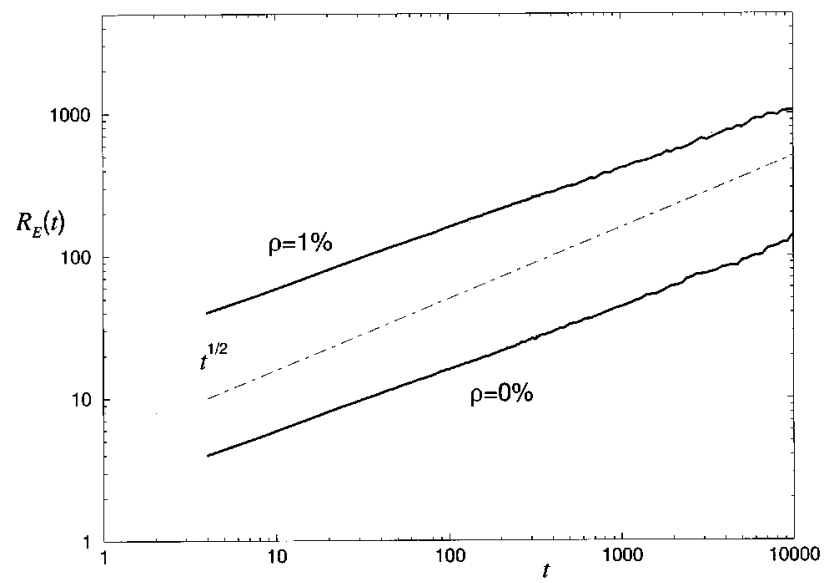

FIG. 1. Double-logarithmic plot of the average linear length scale, $R_{E}(t)$ in Eq. (2), versus $t$ for both the pure $(\rho=0)$ and the dilute $(\rho=0.01)$ Ising model. The top curve is displaced for clarity. The dash-dotted line is $t^{1 / 2}$, the ideal Lifshitz-Allen-Cahn growth law. The temperature is $T / T_{c}=0.8$ and the system size is $N=200^{2}$. Time is measured in units of MCS per lattice site.

choices made for $N_{-}(=4)$ and $N_{+}(=100)$ do not qualitatively affect the results. In the thermodynamic limit, the equilibrium global order parameter, defined as the symmetry-broken one-domain state, is $\Psi(T)$, and in the long-time limit the relation $\Psi_{\text {loc }}(t \rightarrow \infty) \simeq \Psi(T)$ holds. That these are not strictly the same is due to the introduction of the lower cutoff, $N_{-}$, which is necessary in order to distinguish domains from spin excitations. As a second measure of the local order within the ordering domains, we have calculated the domain vacancy concentration in analogy with Eq. (3), i.e.,

$$
\rho_{\text {loc }}(t)=1-\overline{\left(\sum_{k}^{M} N_{k}\right)^{-1} \sum_{k=1}^{M} \sum_{i_{k}=1}^{N_{k}} n_{i_{k}} .}
$$

In Fig. 1 the time dependence of the linear length scale, $R_{E}(t)$, is shown as calculated from Eq. (2). For comparison, the analogous growth curve for the pure system $(\rho=0)$ is included in the plot. It is seen that both curves follow an algebraic growth law, $R(t) \sim A t^{n}$, over several decades. The

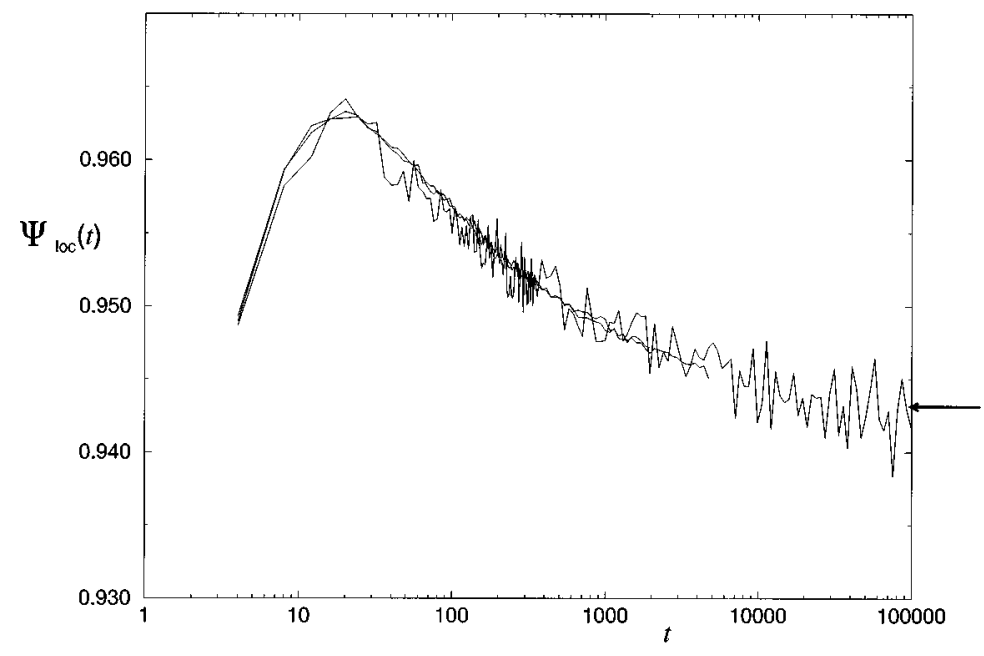

FIG. 2. Semilogarithmic plot of the local domain order parameter, $\Psi_{\text {loc }}(t)$, as defined in Eq. (3). The arrow on the right indicates the equilibrium value, $\Psi(T)$, for a system of size $N=200^{2}$. Results for three system sizes have been superimposed: $N=200^{2}, N=500^{2}$, and $N=1000^{2}$. There is no significant size dependence. The longest run is for $N=200^{2}$ and only a single quench which explains the large fluctuations. The quench temperature is $T / T_{c}=0.8$. Time is measured in units of MCS per lattice site. 


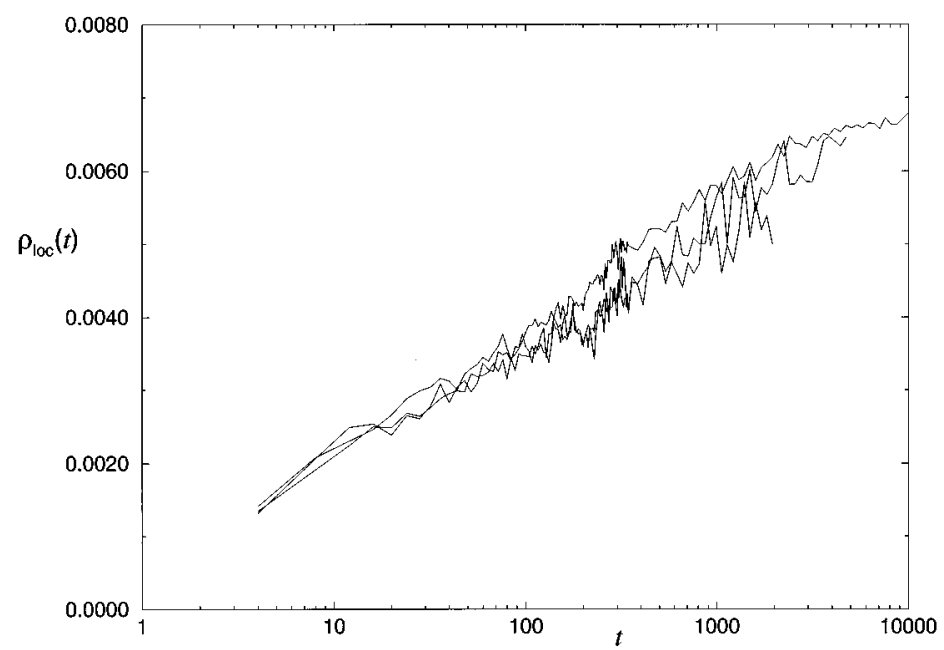

FIG. 3. Semilogarithmic plot of the local domain vacancy concentration, $\rho_{\text {loc }}(t)$, as defined in Eq. (4). The local vacancy concentration is monotonically increasing. The system sizes are as in Fig. 2. Time is measured in units of MCS per lattice site.

growth exponent, $n$, is slightly less than the expected value $1 / 2$ [1] but is apparently the same for both systems. Hence, the growth of the domains is monotonically increasing and the growth exponent is not affected by the introduction of the vacancies. The amplitude, $A$, however, is different. This is not surprising, since the amplitude is a nonuniversal quantity and is therefore expected to depend on both the quench temperature and microscopic details.

In Fig. 2, the local domain order parameter, $\Psi_{\text {loc }}(t)$, as defined in Eq. (3), is monitored as a function of time for three different system sizes. There are some fluctuations in the data, but no significant system-size dependence. The local domain order is obviously a nonmonotonically varying function of time exhibiting a distinct maximum. Furthermore, the maximum is above the equilibrium value of the local order parameter. This phenomenon has been termed overshooting, and it has previously been observed in several different systems $[3,4,6]$ including the pure Ising model [5]. As previously mentioned, the quench is into the ferromagnetically ordered phase where the vacancies in equilibrium are dispersed in the system. Possible time-dependent deviations from the equilibrium distribution of the vacancies can be gauged from the domain vacancy concentration, $\rho_{\text {loc }}(t)$ in Eq. (4), as shown in Fig. 3. This figure demonstrates that for the times shown, $\rho_{\text {loc }}(t)$ is less than the equilibrium value, $\rho=0.01$, and it increases slowly towards this value. $\rho_{\text {loc }}(t)$ loses its physical meaning at very early times where the concept of an ordered domain is lost. Hence, we have found that the domains are transiently depleted in vacancies that are accumulating at the domain boundaries. Only at later times do the vacancies, along with the annealing of the domain pattern, dissolve in the ordered domains again. The depletion of vacancies in the domains also contributes to the overshooting in $\Psi_{\text {loc }}(t)$.
In conclusion, we have studied the domain growth kinetics in an annealed site-diluted Ising system over a wide time span, including the early and intermediate time regimes. As expected for a system with a nonconserved order parameter, the linear domain size grows at late times according to $t^{1 / 2}$ as predicted by the classical Lifshitz-Allen-Cahn theory [1]. The slight deviation in the growth exponent from the expected $n=1 / 2$, seen in Fig. 1 , is most likely due to the fact that when the local order is higher than the equilibrium order, the domain energy is lower. Therefore, not all of the excess energy is located in the boundaries and the assumptions leading to Eq. (2) are not fully correct and lead to an underestimate of $n$. At earlier times, the local ordering within the domains transiently overshoots its equilibrium value, and concomitantly, the vacancies are expelled from the domains. The effect of diffusing vacancies on ordering dynamics has previously been investigated by Srolovitz and Hassold [9] using the same model as here, Eq. (1). Although these authors calculated the local concentration of vacancies in the various parts of the system, their data did not reveal the depletion effect of vacancies in the Ising domains because their quench temperature was very low $\left(T / T_{c}=0.04\right)$. At this low temperature, the equilibrium state of the system corresponds to phase separation between a dilute ferromagnetic phase and a disordered, vacancy-rich phase. Still, their results agree well with the results of both Refs. $[4,5]$, in the sense that it was there shown that the overshooting effect is decreasing with decreasing temperature, which, in turn, has been interpreted as a signal suggesting that the overshooting effect is of entropic origin.

This work was supported by the Danish Natural Science Research Council and partially supported by the MRL Program of the National Science Research Foundation under Contract No. DMR-9123048.
[1] J. D. Gunton, M. San Miguel, and P. S. Sahni, in Phase Transitions and Critical Phenomena, edited by C. Domb and J. L. Lebowitz, (Academic Press, New York, 1983), Vol. 8, p. 267.

[2] A. Bray, Adv. Phys. 43, 357 (1994).
[3] C. Jeppesen and O. G. Mouritsen, Phys. Rev. B 47, 14724 (1993).

[4] H. Gilhøj, C. Jeppesen, and O. G. Mouritsen, Phys. Rev. E 52, 1465 (1995). 
[5] H. Gilhøj, C. Jeppesen, and O. G. Mouritsen, Phys. Rev. Lett. 75, 3305 (1995).

[6] B. Park, G. B. Stephenson, S. M. Allen, and K. F. Ludwig, Jr., Phys. Rev. Lett. 68, 1742 (1992).

[7] R. B. Stinchcombe, in Phase Transitions and Critical Phenom- ena, edited by C. Domb and J. L. Lebowitz (Academic Press, New York, 1983), Vol. 7.

[8] K. Binder and D. Stauffer, Phys. Rev. Lett. 33, 1006 (1974).

[9] D. J. Srolovitz and G. N. Hassold, Phys. Rev. B 35, 6902 (1987). 